\title{
Parâmetros genéticos para idade ao primeiro parto estimados por diferentes modelos para rebanhos da raça Nelore
}

\author{
Genetic parameters for age at first calving estimated by different models for Nellore breed herds
}

\author{
Arione Augusti Boligon ${ }^{\mathrm{I}}$ Pedro Alejandro VozziI ${ }^{\mathrm{II}}$ Jeferson NomeliniI \\ Paulo Roberto Nogara Rorato ${ }^{\text {III }}$ Luiz Antonio Framartino BezerraII \\ Raysildo Barbosa Lôbo II
}

\section{RESUMO}

Com objetivo de estimar parâmetros genéticos $e$ estudar a utilização de diferentes efeitos em avaliações genéticas para idade ao primeiro parto (IPP) por diferentes modelos, foram utilizados registros de IPP de animais da raça Nelore, nascidos entre os anos de 1990 e 2005. Foram considerados os seguintes modelos (M): M1, incluindo o efeito fixo de GC1 (constituído pelos animais nascidos na mesma fazenda e ano), além da covariável, peso aos 365 dias de idade (efeito linear e quadrático), totalizando 24.263 registros de IPP; M2, considerando os efeitos fixos de GC1, ano e estação de parição, totalizando 59.792 registros de IPP e M3, incluindo os efeitos fixos de GC2 (agrupando os animais nascidos na mesma fazenda, ano e que conceberam no mesmo manejo reprodutivo), ano e estação de parição, totalizando 59.792 registros de IPP. As estimativas dos componentes de variância e herdabilidade e os valores genéticos (VG) foram obtidos pelo método da máxima verossimilhança restrita, com a inclusão da matriz de parentesco disponível. As diferenças esperadas na progênie (DEPs) foram obtidas dividindo os VG por dois. Após a obtenção desses resultados, foram realizadas correlações entre os VG e o ranqueamento das DEPs dos reprodutores para IPP, utilizando-se o procedimento PROC CORR (SAS, 2003). Ao se considerar o ano e a estação de parto nos modelos de análise (M2 e M3), esses produziram um maior $R^{2}$, indicando que tais modelos conseguiram explicar, em maior grau, as diferenças existentes entre os animais para IPP. As herdabilidades estimadas foram de baixa magnitude $(0,14 \mathrm{e}$ 0,15). As correlações entre os VG obtidas por diferentes modelos foram 0,73 (M1 x M2); 0,91 (M2 x M3) e 0,66 (M1 x M3).

Palavras-chave: correlação de rank, herdabilidade, precocidade sexual, raça Nelore.

\begin{abstract}
With the objective of estimate genetic parameters and study the utilization of different effects on the genetic evolution for the age of the first calving (AFC) by different models, they were used AFC records from Nellore breed animals, born from 1990 to 2005. They were considered the following models (M): M1, including the fixed effect of the contemporary group (CG), CG1 (grouping the animals that were born at the same farm and year), besides the co variable, weight at 365 days of age (linear and quadratic effects), totalizing 24,263 records of AFC; M2, considering the effects of CG1, year and season of the calving, totalizing 59,792 records of AFC and M3, including effects CG2 (grouping the animals born at the same farm, year and submitted at the same reproductive management), year and season of calving totalizing 59,792 records of AFC. The components of variance and Genetic Value (GV) were obtained by Restricted Maximun Likelihood Method, with the inclusion for the relationship matrix. The Differences Expected on the Progeny (DEPs) were obtained by dividing the $G V$ by two, after this they were estimated the correlations between $G V$ and ranks of the reproductor's DEPs for AFC, utilizing the procedure CORR (SAS, 2003). While considering year and season of calving in the analysis models (M2 and M3), it was produced a bigger $R^{2}$, indicating that those models could explain, in a larger scale, the existing differences between the animals for AFC. The heritabilities estimated were of low magnitude $(0,14$ and 0,15). The correlations between the GV obtained by the different models were .73 (M1 x M2); .91 (M2 $x$ M3) and .66 (M1 x M3).
\end{abstract}

Key words: heritability, Nellore breed, rank correlation, sexual precocity.

IDepartamento de Zootecnia, Faculdade de Ciências Agrárias e Veterinárias, Universidade Estadual Paulista (FCAV/UNESP). Email: arioneb@bol.com.br. Endereço para correspondência. Rua Caetano Grillo, 40, 14883-354, Jaboticabal, SP, Brasil.

${ }^{I I}$ Departamento de Genética, Faculdade de Medicina de Ribeirão Preto, Universidade de São Paulo (FMRP/USP), Ribeirão Preto, SP, Brasil.

IIIDepartamento de Zootecnia, Universidade Federal de Santa Maria (UFSM), Santa Maria, RS, Brasil. 


\section{INTRODUÇÃO}

A redução nas margens de lucro na cadeia produtiva da carne tem levado à busca de maior eficiência. Neste contexto, as características reprodutivas vêm se destacando nos programas de melhoramento genético, pois fertilidade e precocidade sexual são componentes importantes no aumento da produtividade. Segundo SCWENGBER et al. (2001), deve-se buscar o equilíbrio entre os fatores que determinam a fertilidade, pois essa, aliada à precocidade, eleva a taxa de nascimentos no rebanho, auxiliando no processo de seleção, bem como aumentando o desfrute, resultando em maior rentabilidade.

O perímetro escrotal tem sido bastante utilizado em programas que visam a selecionar para a fertilidade. Todavia, segundo PEREIRA et al. (2002), sua eficácia é questionável, em função da baixa correlação genética com precocidade e fertilidade nas fêmeas. Diante disso, vários autores têm estudado características reprodutivas que se expressam diretamente nas fêmeas, como a idade ao primeiro parto, com objetivo de utilizá-las em programas de melhoramento (LÔBO et al., 2000; VAN MELIS et al., 2001; PEREIRA et al., 2002; GRESSLER, 2004; DIAS et al., 2004; BOLIGON et al., 2007).

A idade com que as novilhas parem pela primeira vez influencia diretamente no desempenho reprodutivo do rebanho (MARTIN et al., 1992), porque fêmeas que parem mais cedo têm maiores chances de produzirem maior número de progênies em relação às fêmeas mais tardias. Segundo SHORT et al. (1994), o procedimento de emprenhar novilhas mais jovens promove o retorno mais rápido do capital investido, além de aumentar a vida reprodutiva e o número de progênies por vaca.

Por apresentarem baixa herdabilidade e serem de difícil mensuração, a seleção para precocidade sexual nas fêmeas não é simples. Além disso, alguns produtores atrasam a entrada das fêmeas na reprodução, determinando uma idade ou um peso para que estas iniciem sua vida reprodutiva (DIAS et al., 2004). Outro fator importante é a utilização de estações de acasalamento de curta duração. Esses fatores ambientais são difíceis de serem considerados nas análises, devido, principalmente, à falta dessas informações nos bancos de dados zootécnicos. Diante disso, MERCADANTE et al. (2000) sugeriram a necessidade do estudo de modelos que considerem o maior número de efeitos que influenciam a idade ao primeiro parto, com o objetivo de se reduzirem erros nos resultados das avaliações genéticas de reprodutores, promovendo resultados mais rápidos na seleção dos rebanhos.
Os objetivos deste trabalho foram estimar parâmetros genéticos para idade ao primeiro parto em novilhas da raça Nelore, utilizando-se diferentes modelos e estruturas de dados, e comparar, através de correlação, o ranqueamento em que os touros se encontram para as diferenças esperadas na progênie.

\section{MATERIAL E MÉTODOS}

Foram utilizados registros de idade ao primeiro parto (IPP) de fêmeas participantes do Programa de Melhoramento Genético da Raça Nelore (PMGRN), que pariram entre os anos de 1990 e 2005. O arquivo original continha informações de 60.421 fêmeas, filhas de 29.425 matrizes e 22.795 touros.

Na formação do arquivo de trabalho, os animais foram organizados em dois grupos de contemporâneos (GC), sendo o GC1 constituído pelos animais nascidos na mesma fazenda e no mesmo ano, totalizando 1.224 grupos distintos, e o GC2 reunindo os animais nascidos na mesma fazenda, no mesmo ano e que receberam o mesmo manejo reprodutivo (MR), totalizando 2.329 grupos distintos. A variável MR também foi dividida em dois grupos: MR1, reunindo as fêmeas que pariram até os 30 meses de idade, portanto, aquelas que conceberam em sua primeira estação de monta, e MR0, incluindo as fêmeas que não pariram até os 30 meses de idade, assumindo que estas também tinham sido expostas, juntamente com as do MR1. Foram consideradas duas estações de nascimento (EN), sendo a EN1 constituída pelos animais nascidos entre janeiro e junho e a EN2 constituída pelos animais nascidos entre julho e dezembro.

As variáveis a serem incluídas nos modelos para estudos genéticos foram identificadas pela significância estatística de seus efeitos sobre IPP, através de uma análise de variâncias, pelo método dos quadrados mínimos, utilizando-se o procedimento GLM do SAS (SAS, 2003). A partir desses resultados, foram definidos os três modelos animais (M) a seguir descritos:

M1: considerou como fixos os efeitos de GC1, além da covariável peso aos 365 dias de idade, efeitos linear e quadrático e, como aleatórios, os efeitos genéticos aditivos diretos do animal e o residual, para um total de 24.263 registros de IPP;

M2: considerou como fixos os efeitos de GC1, ano e estação de parição e, como aleatórios, os efeitos genéticos aditivos diretos do animal e o residual, para um total de 59.792 registros de IPP;

M3: considerou como fixos os efeitos de GC2, ano e estação de parição e, como aleatórios, os efeitos genéticos aditivos diretos do animal e o residual, para um total de 59.792 registros de IPP. 
Os componentes de (co)variância utilizados para estimar os coeficientes de herdabilidade e predizer os valores genéticos (VG) foram obtidos pelo Método da Máxima Verossimilhança Restrita, adotando-se um modelo animal, com a inclusão da matriz de parentesco disponível entre os animais fornecedores de informações e seus antepassados, em três análises unicaracterísticas. O modelo misto geral pode ser representado de forma matricial como:

em que:

$$
y=X \beta+Z a+e
$$

$\mathrm{y}=$ vetor de variáveis dependentes;

$\beta=$ vetor de efeitos fixos;

$a=$ vetor de valores genéticos aditivos dos animais;

$e=$ vetor de efeitos residuais;

$X$ e $\mathrm{Z}=$ matrizes de incidência respectivas para cada efeito.

As pressuposições em relação aos componentes do modelo são:

$\mathrm{E}(y)=X \beta, E(a)=0$ e $E(e)=0$

$\operatorname{Var}\left[\begin{array}{l}a \\ e\end{array}\right]=\left[\begin{array}{ll}G & 0 \\ 0 & R\end{array}\right]$

em que:

$\mathrm{G}=$ matriz de variância genética aditiva, obtida por $\mathrm{A} \sigma_{a}^{2}$;

$\mathrm{A}=$ matriz de parentesco;

$R=$ matriz de variância residual, obtida por $\mathrm{I} \sigma_{e}^{2}$;

$\mathrm{I}=$ matriz identidade;

$\sigma_{e}^{2}=$ variância genética aditiva direta;

$\sigma_{e}^{2}=$ variância residual.

Para a análise 1 foi utilizado, como modelo animal, M1, acima descrito, acrescido dos efeitos aleatórios genético aditivo direto do animal e residual. O mesmo procedimento foi adotado para M2 e M3. O aplicativo computacional utilizado foi o MTDFREML (BOLDMAN et al., 1995). O critério de convergência para as análises com o MTDFREML foi $10^{-6}$. Após cada convergência, o programa era reiniciado, tendo como valores iniciais as estimativas da análise anterior, até -2 Log não se modificar por três análises, então, o procedimento era finalizado.

As correlações genéticas entre os VG da característica de IPP sob diferentes modelos foram estimadas pelo procedimento PROC CORR, Pearson (SAS, 2003), em que a estimativa de correlação entre valores genéticos é a própria definição de correlação genética (VAN VLECK et al., 1987). As diferenças esperadas nas progênies (DEP) dos animais foram obtidas dividindo-se os VG por dois. Pelo procedimento CORR, Spearman (SAS, 2003), foram estimadas as correlações entre os ranqueamentos das DEP para os animais avaliados.

\section{RESULTADOS E DISCUSSÃO}

A média para IPP obtida neste trabalho foi de 36,52 meses, estando próxima às relatadas por PEREIRA et al. (2000), DIAS et al. (2004) e BOLIGON et al. (2007), as quais foram de 35,67; 34,6 e 36,21 meses, respectivamente, para fêmeas da raça Nelore. Por outro lado, LÔBO et al. (2000), trabalhando com 34.037 fêmeas, relataram IPP médias de 40,20 meses.

Os resultados das análises de variância realizadas para os diferentes modelos encontram-se na tabela 1. Considerando o M1, verifica-se que o efeito do peso aos 365 dias de idade não foi significativo sobre IPP, justificando a sua não-inclusão nos M2 e M3. O maior coeficiente de determinação $\left(R^{2}\right)$ foi obtido para o M3 $(0,80)$, seguido pelo M2 $(0,76)$, mostrando que, com a inclusão do ano e da estação de parição no modelo de análise (M2 e M3), esses foram capazes de explicar a maior parte das diferenças existentes entre os animais quanto à idade ao primeiro parto.

As variâncias estimadas em cada modelo indicam que, com a inclusão da covariável peso aos 365 dias de idade (M1), obteve-se maior variabilidade para IPP. Esse resultado nos permite concluir que o peso aos 356 dias de idade foi responsável por considerável variação genética, ambiental e fenotípica nos rebanhos estudados, sendo importante considerálo nos modelos de avaliações genéticas de fêmeas da raça Nelore.

Tabela 1 - Análises de variância para a idade ao primeiro parto sob diferentes modelos.

\begin{tabular}{|c|c|c|c|c|}
\hline Fontes de variação & $\mathrm{gl}$ & $\begin{array}{l}\text { Quadrado } \\
\text { médio }\end{array}$ & $\mathrm{F}$ & $\operatorname{Pr}>F$ \\
\hline \multicolumn{5}{|c|}{ Modelo 1} \\
\hline GC1 & 807 & 186,0489 & 11,20 & 0,0001 \\
\hline P365 (linear) & 1 & 111,2116 & 6,69 & 0,0097 \\
\hline P365 (quadrático) & 1 & 412,4752 & 24,83 & 0,0001 \\
\hline Resíduo & 23.453 & 16,6137 & & \\
\hline $\mathrm{R}^{2}$ & 0,58 & & & \\
\hline \multicolumn{5}{|c|}{ Modelo 2} \\
\hline GC1 & 1.223 & 1091,337 & 151,53 & 0,0001 \\
\hline Ano & 15 & 51259,911 & 7117,34 & 0,0001 \\
\hline Estação & 1 & 142860,247 & 19835,9 & 0,0001 \\
\hline Resíduo & 58.732 & 7,202 & & \\
\hline $\mathrm{R}^{2}$ & 0,76 & & & \\
\hline \multicolumn{5}{|c|}{ Modelo 3} \\
\hline GC2 & 2.328 & 599,570 & 95,47 & 0,0001 \\
\hline Ano & 15 & 28050,051 & 4466,51 & 0,0001 \\
\hline Estação & 1 & 70917,672 & 11292,5 & 0,0001 \\
\hline Resíduo & 57.627 & 6,280 & & \\
\hline $\mathrm{R}^{2}$ & 0,80 & & & \\
\hline
\end{tabular}


As herdabilidades estimadas para IPP nos diferentes modelos foram consideradas baixas (Tabela 2), podendo ser explicadas, em parte, devido a uma porção da variação existente na característica não ser estimada, pois somente as fêmeas que pariram são consideradas nas análises. Dessa forma, mesmo utilizando-se um arquivo de genealogia bastante completo, muitas vezes não é possível obter a real variabilidade genética existente na população.

Estimativas de herdabilidade de baixa magnitude também foram relatadas por DIAS et al. (2004), as quais variaram de 0,08 a 0,16 , para diferentes modelos analisados. Tais autores enfatizam que a curta duração da estação de monta permitida aos animais pode estar influenciando tais resultados. Além disso, os mesmos autores sugerem que, se fosse permitido que as fêmeas entrassem em reprodução durante todo o ano, as diferenças entre os animais, provavelmente, seriam notadas.

No geral, os resultados obtidos neste estudo encontram-se próximos aos relatados na literatura para esta mesma raça, os quais variam de 0,10 a 0,19 (PEREIRA et al., 2000; SCHWENGBER et al., 2001; PEREIRA et al., 2002 e BOLIGON et al., 2007). Por outro lado, VAN MELIS et al. (2001) e PEREIRA et al. (2002) relataram estimativas de herdabilidade para IPP praticamente nulas, ou seja, 0,01 e 0,02, respectivamente.

É importante salientar que diferentes amostras e modelos causam disparidades nas estimativas de parâmetros genéticos para características reprodutivas. Isso ocorre porque a idade ao primeiro parto é bastante dependente do manejo reprodutivo e da idade em que as fêmeas são desafiadas à concepção. Assim, dependendo da forma que tais efeitos são considerados ou não nos modelos, as estimativas obtidas podem sofrer grandes variações.

Na tabela 3, encontram-se descritos os resultados das correlações genéticas, classificação (rank) dos touros avaliados através das DEPs e médias

\begin{tabular}{lcccc} 
Tabela 2 & $-\begin{array}{l}\text { Estimativas de componentes de variância e } \\
\text { herdabilidade para idade ao primeiro parto sob } \\
\text { diferentes modelos de análise, para fêmeas da raça } \\
\text { Nelore. }\end{array}$ \\
\hline Modelos & $\sigma^{2}{ }_{\mathrm{a}}$ & $\sigma^{2}{ }_{\mathrm{e}}$ & $\sigma^{2}{ }_{\mathrm{p}}$ & $\mathrm{h}^{2}$ \\
\hline 1 & 2.5059 & 14.2759 & 16.7818 & 0,15 \\
2 & 1.0479 & 6.2292 & 7.2771 & 0,14 \\
3 & 1.6182 & 9.9404 & 11.5586 & 0,14 \\
\hline
\end{tabular}

variância genética aditiva $\left(\sigma_{\mathrm{a}}^{2}\right)$; variância ambiental $\left(\sigma_{\mathrm{e}}^{2}\right)$; variância fenotípica $\left(\sigma_{\mathrm{p}}^{2}\right)$; herdabilidade $\left(\mathrm{h}^{2}\right)$.
Tabela 3 - Estimativas de correlação genética (acima da diagonal), de classificação (rank) dos touros avaliados (abaixo da diagonal) e médias das DEPs (diagonal principal) para idade ao primeiro parto (IPP) de animais da raça Nelore.

\begin{tabular}{llcl}
\hline DEPs & Modelo 1 & Modelo 2 & Modelo 3 \\
\hline Modelo 1 & 0,0957 & $0,73^{*}$ & $0,66^{*}$ \\
Modelo 2 & $0,57^{*}$ & 0,1516 & $0,91^{*}$ \\
Modelo 3 & $0,56^{*}$ & $0,83^{*}$ & 0,1415 \\
\hline
\end{tabular}

${ }^{*} \mathrm{P}<0,0001$

das DEPs para característica de IPP, avaliada sob diferentes modelos. Podem ser observadas correlações genéticas próximas a unidade $(0,91)$ quando comparouse o M2 com o M3, indicando que, independentemente da escolha de um desses modelos, os resultados dos VG seriam semelhantes. Por outro lado, quando foram comparados o M1 com os M2 e M3, obteve-se correlações genéticas de 0,73 e 0,66 , respectivamente. Este resultado sugere que as três expressões de IPP estão sob a influência dos mesmos genes com mesma direção e sentido, porém com magnitudes diferentes (médias a altas).

Diferenças pequenas foram constatadas entre as correlações de ranqueamentos dos touros classificados para as DEPs, quando foram comparados M2 e M3, sugerindo haver pouca diferença na classificação dos animais nesse caso. Verificam-se também correlações inferiores quando se compara o ranqueamento dos touros classificados para as DEPs entre de M1 com os demais. Isso indica grandes diferenças na classificação dos touros quando optarse-ia por estimar DEPs utilizando-se o M1 em relação aos demais, o que deve ser considerado nos programas de seleção que visam a reduzir a IPP, pois pode resultar em escolhas inadequadas de reprodutores.

As DEPs médias obtidas foram positivas e próximas a zero (Tabela 3), sugerindo ser possível melhorar esta característica no rebanho avaliado se forem identificados os reprodutores que apresentem menores DEPs para IPP, utilizando-se estes com maior intensidade nos rebanhos.

Na tabela 4, apresentam-se os graus de nãocoincidência entre a classificação dos reprodutores das DEPs para característica de IPP, considerando-se os diferentes modelos estudados. Os resultados evidenciam que, ao se estimar DEPs com o M1, os touros selecionados como melhores não seriam, em sua maioria, os mesmos se tais valores fossem estimados pelos M2 e M3.

Ciência Rural, v.38, n.2, mar-abr, 2008. 
Tabela 4 - Classificação dos 10 primeiros reprodutores, de acordo com a DEP para IPP sob diferentes modelos.

\begin{tabular}{cccc}
\hline & Modelo1 & Modelo 2 & Modelo3 \\
\hline Posição & Identidade do animal & Posição & Posição \\
$1^{\circ}$ & $\mathrm{AB}$ & $8^{\circ}$ & $9^{\circ}$ \\
$2^{\circ}$ & $\mathrm{AC}$ & $103^{\circ}$ & $97^{\circ}$ \\
$3^{\circ}$ & $\mathrm{AD}$ & $4^{\circ}$ & $3^{\circ}$ \\
$4^{\circ}$ & $\mathrm{AE}$ & $22^{\circ}$ & $19^{\circ}$ \\
$5^{\circ}$ & $\mathrm{AF}$ & $5^{\circ}$ & $5^{\circ}$ \\
$6^{\circ}$ & $\mathrm{BG}$ & $35^{\circ}$ & $41^{\circ}$ \\
$7^{\circ}$ & $\mathrm{BH}$ & $1^{\circ}$ & $1^{\circ}$ \\
$8^{\circ}$ & $\mathrm{BI}$ & $3^{\circ}$ & $4^{\circ}$ \\
$9^{\circ}$ & $\mathrm{BJ}$ & $52^{\circ}$ & $56^{\circ}$ \\
$10^{\circ}$ & $\mathrm{BK}$ & $6^{\circ}$ & $8^{\circ}$ \\
\hline
\end{tabular}

Comparando os 10 melhores reprodutores selecionados para os M2 e M3 (Tabela 4), pode-se afirmar que, em grande parte, a classificação dos mesmos não sofreria maiores distinções. Mesmo assim, a classificação dos indivíduos por um ou outro critério pode trazer conseqüências diferentes, a longo prazo, nos programas de melhoramento.

Esses resultados de não-coincidência indicam que $50 \%$ ou mais dos animais que seriam utilizados por um critério de seleção não seriam pelo outro, confirmando o grande impacto que tem a escolha adequada de fontes de variação a serem incluídas nos modelos de análise. Além disso, cabe destacar a importância de se compararem somente indivíduos avaliados com o mesmo modelo.

\section{CONCLUSÕES}

Apesar de as estimativas de herdabilidade obtidas para idade ao primeiro parto serem consideradas de baixa magnitude, elas indicam a possibilidade de inclusão desta característica como forma de auxiliar nos programas de seleção.

Os coeficientes de correlação estimados sugerem que, dependendo do modelo a ser utilizado, poderá ocorrer a escolha de animais diferentes. Dessa forma, torna-se importante a escolha do modelo de análise adequado que considere ao máximo os efeitos que influenciam a característica de idade ao primeiro parto nos rebanhos avaliados.

Os resultados obtidos sugerem diferenças nas correlações genéticas e na classificação dos reprodutores quando se utiliza o M1, em comparação com os M2 e 3. Sendo assim, sugere-se a utilização dos efeitos de ano e estação de parição, além do manejo reprodutivo nos modelos de avaliação para característica de idade ao primeiro parto em rebanhos da raça Nelore.

\section{AGRADECIMENTO}

Ao Programa de Melhoramento Genético da Raça Nelore (PMGRN), pelo fornecimento das informações utilizadas neste estudo e à Fundação de Amparo à Pesquisa do Estado de São Paulo (FAPESP), pela concessão de bolsa ao pesquisador Boligon.

\section{REFERÊNCIAS}

BOLDMAN, K.G. et al. A manual for use for MTDFREML. A set of programs to obtain estimates of variance and covariance (DRAFT). Lincoln: Department of Agricultural/Agricultural Research Service, 1995. 120p.

BOLIGON, A.A. et al. Correlações genéticas entre medidas de perímetro escrotal e características produtivas e reprodutivas de fêmeas da raça Nelore. Revista Brasileira de Zootecnia, v.36, n.3, p.565-571, 2007.

DIAS, L.T. et al. Estimativas de herdabilidade para idade ao primeiro parto de novilhas da raça Nelore. Revista Brasileira de Zootecnia, v.33, n.1, p.97-102, 2004.

LÔBO, R.N.B. et al. Average estimates of genetics parameters for beef and dairy cattle in tropical regions. Animal Breeding Abstract, v.68, p.433-461, 2000.

GRESSLER, S.L. Fatores ambientais e genéticos do perímetro escrotal e da idade ao primeiro parto em novilhas Nelore desafiadas tradicional ou precocemente. 2004. 139f. Tese (Doutorado em Zootecnia) - Universidade Federal de Minas Gerais.

MARTIN, L.C. et al. Genetic effects on beef heifer puberty and subsequent reproduction. Journal of Animal Science, v.70, p.4006-4017, 1992.

MERCADANTE, M.E.Z. et al. Estimativas de (co) variâncias entre características de reprodução e de crescimento e fêmeas de um rebanho Nelore. Revista Brasileira de Zootecnia, v.29, n.4, p.997-1004, 2000.

PEREIRA, E. et al. Correlação entre perímetro escrotal e algumas características reprodutivas na raça Nelore. Revista Brasileira de Zootecnia, v.29, n.6, p.1676-1683, 2000.

PEREIRA, E. et al. Análise genética de características reprodutivas na raça Nelore. Pesquisa Agropecuária Brasileira, v.37, n.5, p.703-708, 2002.

SAS Institute, SAS (Statistical Analysis System). User's Guide. Cary, NC, 2003. 129p.

SCHWENGBER, E.B. et al. Produtividade acumulada como critério de seleção em fêmeas da raça Nelore. Ciência Rural, v.31, n.3, p.483-486, 2001.

SHORT, R.Y. et al. Breeding heifers at one year of age: biological and economic considerations. In: FIELDS, M.J.; SAND, R.S. (Eds.). Factors affecting calf crop. London: CRC, 1994. p.55-68.

VAN MELIS, M.H. et al. Efeito das classes a desmama e herdabilidade para idade ao primeiro parto em novilhas da raça Nelore. In: REUNIÃO ANUAL DA SOCIEDADE BRASILEIRA DE ZOOTECNIA, 38., 2001, Piracicaba. Anais... Piracicaba: Sociedade Brasileira de Zootecnia, 2001. p.524-526.

VAN VLECK, L.D. et al. Genetic for the animal breeding. New York: Freeman, 1987. 391p. 